\title{
Analysis and Control of a Gait of Snake Robot
}

\author{
Non-member Pavel Prautsch (Tokyo Institute of Technology) \\ Member Tsutomu Mita (Tokyo Institute of Technology) \\ Member Tetsuya Iwasaki (Tokyo Institute of Technology)
}

In this paper, we develop a model of a snake-like robot consisting of $n$ rigid links and propose several control strategies to achieve smooth undulative locomotion and position control. The model is based on nonholonomic velocity conditions that arise from non-slipping assumption about the wheels. Singular postures of the robot are analyzed and they are taken in account while deriving the control law. The control strategies are based on Lyapunov method and on constraining the trace of the snake's head into some kind of serpenoid trace, respectively. The later method is also applied for a simple tracking control method.

Keywords: snake robot, nonholonomic system, Lyapunov method, variable constraint control

\section{Introduction}

Unique and interesting gait of snakes allows them to move through an ill-conditioned environment. Therefore, investigating snake's gait by making a robot is reasonable and shall result in design of new walking and transporting mechanisms.

Hirose $^{(1)}$ of our university has investigated snake robots and natural snakes. He has made several snake robots, where he modeled the snake by a link mechanism with no side slip. As the control inputs, he used sinusoidal functions and tuned the amplitude, frequency and phase differences to drive the robot to a certain direction. However, the positioning control of the snake's head was shown to be difficult. He also described the trace of body of natural snakes as what he called a 'serpenoid curve' which guarantees that the distribution of a strain in the muscle becomes a smooth function.

Comparing to this pioneering research work, the first steps of our research are as follows.

1. Design a snake robot which can creep forward and apply the modern control theory to realize a robot with a position control. Check whether the trace of the body can be approximated by the serpenoid function.

2. Derive a control method which can create an exact serpenoid trace if any, and see what happens with the magnitude and distribution of the control inputs.

3. Using these results, find the best model of snake robots which uses the least energy to move.

In the sequel, we will use the following abbreviations unless confusion may occur

$$
\begin{array}{ll}
\mathrm{C} \theta=\cos \theta, \mathrm{S} \theta=\sin \theta, & \mathrm{C}_{i}=\cos \theta_{i}, \mathrm{~S}_{i}=\sin \theta_{i}, \\
\mathrm{C}_{i j}=\cos \left(\theta_{i}-\theta_{j}\right), & \mathrm{S}_{i j}=\sin \left(\theta_{i}-\theta_{j}\right) .
\end{array}
$$

\section{Model of the snake robot}

Mechanical structure of the snake robot is depicted in Fig. 1. The robot consists of $n$ rigid links and simplifies the robot used by Hirose ${ }^{(1)}$ and is different from

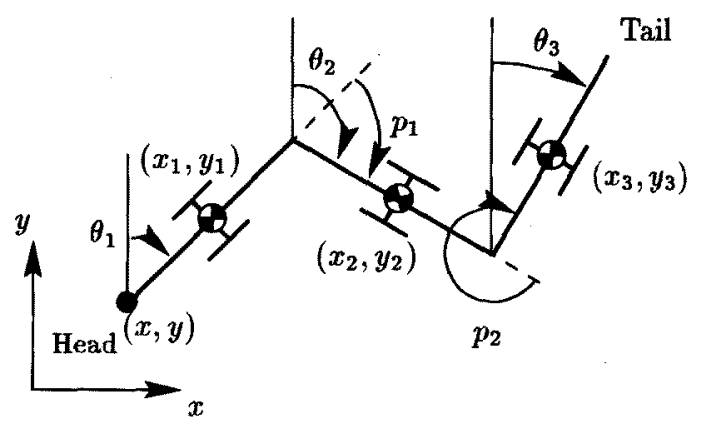

Fig. 1. $n$-link model of the snake robot

the one investigated by Ostrowski and Burdick ${ }^{(2)}$ which has active steering of the wheel axes.

Length $2 l$ and weight $m$ are the same for each link and distribution of the weight is assumed to be uniform. As a result, the COM (center of mass) of each link is placed at the middle of the link.

For each link $\left(x_{i}, y_{i}\right)$ and $\theta_{i}, i=1 \sim n$ represent the position of its COM and absolute angle measured clockwise from $y$ axis. We also denote the position of the head of the robot by $(x, y)$. Moreover, we call $p_{i}=\theta_{i+1}-\theta_{i}(i=1 \sim n-1)$ the relative angle which is controlled by input torques $u \in R^{n-1}$. Finally, a fixed wheel axis with two passive wheels is attached to each link at its COM and thus prevents the COM of each link from going sideways.

From Fig. 1, the position and velocity of each COM are given by

$$
\begin{aligned}
& x_{i}=x+2 l \Sigma_{j=1}^{i-1} \mathrm{~S} \theta_{j}+l \mathrm{~S} \theta_{i}, \\
& y_{i}=y+2 l \Sigma_{j=1}^{i-1} \mathrm{C} \theta_{j}+l \mathrm{C} \theta_{i}
\end{aligned}
$$

and

$$
\begin{aligned}
& \dot{x}_{i}=\dot{x}+2 l \Sigma_{j=1}^{i-1} \dot{\theta}_{j} \mathrm{C} \theta_{j}+l \dot{\theta}_{i} \mathrm{C} \theta_{i} \\
& \dot{y}_{i}=\dot{y}-2 l \Sigma_{j=1}^{i-1} \dot{\theta}_{j} \mathrm{~S} \theta_{j}-l \dot{\theta}_{i} \mathrm{~S} \theta_{i} .
\end{aligned}
$$


From the condition that the wheels cannot slip sideways, the velocity constraints of each link are given by

$$
\dot{x}_{i} \mathrm{C} \theta_{i}-\dot{y}_{i} \mathrm{~S} \theta_{i}=0 \quad: i=1 \sim n . \cdots \cdots \ldots \ldots
$$

Substituting (2) into (3) leads to

$$
l \dot{\theta}_{i}+2 l \Sigma_{j=1}^{i-1} \dot{\theta}_{j} \mathrm{C}_{i j}+\mathrm{C} \theta_{i} \dot{x}-\mathrm{S} \theta_{i} \dot{y}=0 \quad: i=1 \sim n,
$$

or, equivalently, to

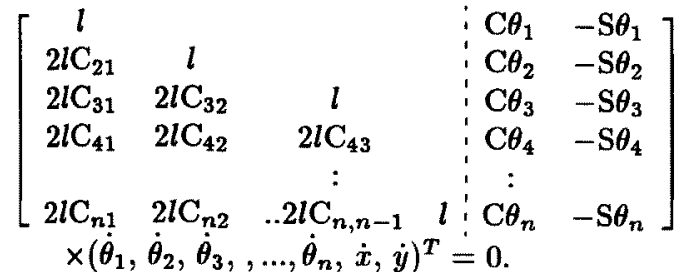

If we write this constraint as

$\left(F_{A},-F_{B}\right)\left[\begin{array}{c}\dot{\theta} \\ \dot{w}\end{array}\right]=0: \theta=\left[\begin{array}{c}\theta_{1} \\ \theta_{2} \\ \vdots \\ \theta_{n}\end{array}\right], w=\left[\begin{array}{c}x \\ y\end{array}\right]$,

since $\left|F_{A}\right| \neq 0,(4)$ can be represented as

$$
\dot{\theta}=F(\theta) \dot{w}: \quad F=F_{A}^{-1} F_{B} .
$$

Note that

$$
\begin{aligned}
& p=E^{T} \theta \quad: p=\left(p_{1}, p_{2}, \ldots, p_{n-1}\right)^{T} \\
& E^{T}=\left[\begin{array}{lllll}
-1 & 1 & & & \\
& & : & & \\
& & -1 & 1
\end{array}\right](n-1 \times n)
\end{aligned}
$$

holds between the absolute angle and the relative angle, and thus the velocity constraint in terms of the relative angle is expressed by

$$
\dot{p}=E^{T} F \dot{w} \text {. }
$$

The purposes of this study can be expressed as to control the final position of $w$ using $u=\dot{p}$ and to investigate whether the body of the robot undulates properly.

This problem is different from ordinary nonholonomic control problems in which $w$ and $\theta$ are controlled by choosing the translational and angular velocity of the head, e.g. the control of trailers ${ }^{(3)}$. Here, the propulsion force arises from a change of the internal shape of the robot. The main technical difficulty is how to avoid the singular posture of the robot which occurs when $E^{T} F$ drops rank.

2.1 Equation of motion We may deal with the problem based on a velocity control. However, it needs an inversion of $E^{T} F$. Therefore, we will derive the equation of motion in order to apply an acceleration based control.

First, consider a motion of an unconstrained mechanism on a ground, which is not necessarily flat. The only difference from a robot arm is that the base of the body is not fixed and every wheel is affected by a viscous friction with the ground.

Using $q=(\theta, w)^{T} \in R^{n+2}$ as the generalized coordinates, the kinetic energy is given by

$$
\begin{aligned}
& \mathcal{T}=\frac{1}{2} \Sigma_{i=1}^{n}\left[m_{i}\left(\dot{x}_{i}^{2}+\dot{y}_{i}^{2}\right)+J_{i} \dot{\theta}_{i}^{2}\right] \\
& =\frac{1}{2}\left(\dot{\theta}^{T}, \dot{w}^{T}\right)\left[\begin{array}{ll}
M_{11} & M_{12} \\
M_{12}^{T} & M_{22}
\end{array}\right]\left[\begin{array}{c}
\dot{\theta} \\
\dot{w}
\end{array}\right] \\
& =\frac{1}{2} \dot{q}^{T} M(\theta) \dot{q},
\end{aligned}
$$

where $M_{11}, M_{21}$ are functions of $\theta$ and $M_{22}=n m I_{2}$. The dissipative energy is assumed to be given by

$$
\begin{aligned}
& \mathcal{D}=\frac{1}{2} \Sigma_{i=1}^{n}\left[D_{x y}\left(\dot{x}_{i}^{2}+\dot{y}_{i}^{2}\right)+D_{\theta} \dot{\theta}_{i}^{2}\right] \\
& +\Sigma_{i=2}^{n} D_{p} \dot{p}_{i}^{2}=\frac{1}{2} \dot{q}^{T} D(\theta) \dot{q},
\end{aligned}
$$

where $D_{x y}$ and $D_{\theta}$ are coefficients of friction of the translational and rotational motion, respectively, of the bottom of the wheel; $D_{p}$ is the coefficient of the friction in the joints.

The potential energy can be written as

$$
\mathcal{U}=n m g\left[X_{C O M} \sin (\alpha)+Y_{C O M} \cos (\beta)\right],
$$

where $g$ is gravity constant, $\left(X_{C O M}, Y_{C O M}\right)$ is position of the COM of the robot (the potential energy is assumed to be zero at $\left.\left(X_{C O M}, Y_{C O M}\right)=(0,0)\right)$ and $\alpha, \beta$ is a gradient of the ground along $x$ and $y$ axis, respectively. This keeps the $z$ axis of the inertial frame always perpendicular to the ground.

From these, the equation of motion without the velocity constraints is given by ${ }^{(4)}$

$$
\begin{aligned}
& \frac{d}{d t}\left(\frac{\partial \mathcal{T}}{\partial \dot{q}}\right)-\frac{\partial \mathcal{T}}{\partial q}+\frac{\partial \mathcal{D}}{\partial \dot{q}}+\frac{\partial \mathcal{U}}{\partial q}-\left[\begin{array}{c}
E u \\
0
\end{array}\right] \\
& =M(\theta)\left[\begin{array}{c}
\ddot{\theta} \\
\ddot{w}
\end{array}\right]+\left[\begin{array}{c}
C_{1}(\dot{\theta}, \theta) \\
C_{2}(\dot{\theta}, \theta)
\end{array}\right] \dot{\theta}+D(\theta)\left[\begin{array}{c}
\dot{\theta} \\
\dot{w}
\end{array}\right] \\
& +U(\theta)-\left[\begin{array}{c}
E u \\
0
\end{array}\right]=0,
\end{aligned}
$$

where the input term was derived from the virtual work principle $u_{\theta}^{T} \delta \theta=u_{p}^{T} \delta p$. The second term represents the centrifugal force and it has the following property of skew symmetry likewise robot arms ${ }^{(5)}$

Lemma 1 Matrix $\dot{M}_{11}-2 C_{1}^{T}$ is skew symmetric. Moreover, $\dot{M}_{12}=C_{2}^{T}$.

It is straightforward to prove this lemma and the proof is therefore omitted.

2.2 Reduce order models In order to incorporate the nonholonomic velocity constraints into the equation motion (9), multiply the Pfaffian constraint,s (5) by $F_{A}^{-1}$ to obtain a more suitable form

$\left[I_{n},-F(\theta)\right]\left[\begin{array}{c}\dot{\theta} \\ \dot{w}\end{array}\right]=A(q) \dot{q}=0: \quad q=\left[\begin{array}{c}\theta \\ w\end{array}\right]$.

Then, use a vector of Lagrange multipliers $\lambda$ to obtain

$$
\begin{aligned}
& M(\theta)\left[\begin{array}{c}
\ddot{\theta} \\
\ddot{w}
\end{array}\right]+\left[\begin{array}{c}
C_{1}(\dot{\theta}, \theta) \\
C_{2}(\dot{\theta}, \theta)
\end{array}\right] \dot{\theta}+D(\theta)\left[\begin{array}{c}
\dot{\theta} \\
\dot{w}
\end{array}\right] \\
& +U(\theta)-\left[\begin{array}{c}
E u \\
0
\end{array}\right]-\left[\begin{array}{c}
I_{n} \\
-F^{T}(\theta)
\end{array}\right] \lambda=0 .
\end{aligned}
$$

To separate the velocity constraint from (11), we multiply (11) by $\left(F^{T}, I_{2}\right)$ from the left and obtain the following reduced order system 

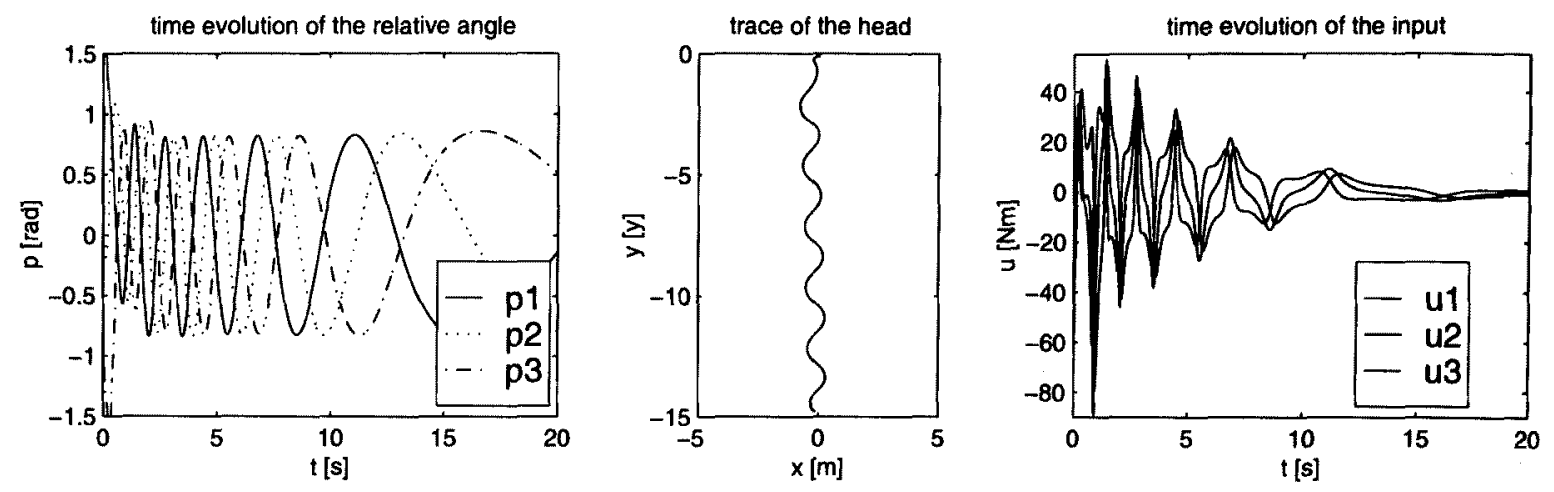

Fig. 2. Simulation results of motion of a four-link snake model

$$
\tilde{M}(\theta) \ddot{w}+\tilde{C}(\theta, \dot{\theta}) \dot{w}+\tilde{D}(\theta) \dot{w}+\tilde{U}(\theta)=F^{T}(\theta) E u
$$

which will be referred to as Model I. Matrices of this model are defined as (from now on we will omit the argument $\theta$ )

$$
\begin{aligned}
& \tilde{M}=\left(F^{T}, I_{2}\right) M\left[\begin{array}{l}
F \\
I_{2}
\end{array}\right]>0, \\
& \tilde{D}=\left(F^{T}, I_{2}\right) D\left[\begin{array}{l}
F \\
I_{2}
\end{array}\right]>0, \\
& \tilde{U}=\left(F^{T}, I_{2}\right) U, \\
& \tilde{C}=\left(F^{T} M_{11}+M_{12}^{T}\right) \dot{F}+F^{T} C_{1} F+C_{2} F .
\end{aligned}
$$

During the derivation, following relations have been used

$$
\dot{\theta}=F \dot{w}, \quad\left[\begin{array}{c}
\ddot{\theta} \\
\ddot{w}
\end{array}\right]=\left[\begin{array}{c}
F \\
I_{2}
\end{array}\right] \ddot{w}+\left[\begin{array}{c}
\dot{F} \\
0
\end{array}\right] \dot{w} .
$$

Model I satisfies the following property.

Lemma 2 Matrix $\dot{\tilde{M}}-2 \tilde{C}$ is skew symmetric.

See Appendix for proof.

We will also show another reduced order model which uses a different set of coordinates.

The translational velocity of the first link $\dot{\eta}_{1}$ is given by

$$
\dot{\eta}_{1}=\dot{x}_{1} \mathrm{~S} \theta_{1}+\dot{y}_{1} \mathrm{C} \theta_{1} .
$$

Utilizing (2), this can be rewritten as

$$
\dot{\eta}_{1}=\dot{x} \mathrm{~S} \theta_{1}+\dot{y} \mathrm{C} \theta_{1}
$$

which describes the velocity of the head along its trace and $\eta_{1}$ is thus a length of the trace of the head. Then, defining a transformation matrix

$$
G=\left[\begin{array}{cc}
-\mathrm{C} \theta_{1} & \mathrm{~S} \theta_{1} \\
\mathrm{~S} \theta_{1} & \mathrm{C} \theta_{1}
\end{array}\right]=G^{T}=G^{-1}
$$

and describing (14) together with $-\dot{x} \mathrm{C} \theta_{1}+\dot{y} \mathrm{~S} \theta_{1}=l \dot{\theta}_{1}$ derived from (4) yields:

$$
G \dot{w}=\left[\begin{array}{c}
l \dot{\theta}_{1} \\
\dot{\eta}_{1}
\end{array}\right]
$$

In addition

$$
F G=F_{A}^{-1} F_{B} G
$$

becomes independent of $\theta_{1}$ as it is easy to see that $F_{B} G$ does not contain $\theta_{1}$ but $p_{1}$.

Then, substituting

$$
\dot{G} \dot{w}+G \ddot{w}=\left[\begin{array}{c}
\ddot{\theta}_{1} \\
\ddot{\eta}_{1}
\end{array}\right]
$$

into (12), we obtain

$\bar{M}\left[\begin{array}{c}l \ddot{\theta}_{1} \\ \ddot{\eta}_{1}\end{array}\right]+\bar{C}\left[\begin{array}{c}l \dot{\theta}_{1} \\ \dot{\eta}_{1}\end{array}\right]+\bar{D}\left[\begin{array}{c}\dot{\theta}_{1} \\ \dot{\eta}_{1}\end{array}\right]+\bar{U}=G F^{T} E u$

which will be referred to as Model II. Here, the matrices $G F^{T} E$ and

$$
\begin{aligned}
& \bar{M}=G \tilde{M} G, \quad \bar{C}=G \tilde{C} G+G \tilde{M} \dot{G} \\
& \bar{D}=G \tilde{D} G, \quad \bar{U}=G \tilde{U}
\end{aligned}
$$

are functions of only $p$ and we have

Lemma 3 Matrix $\dot{\bar{M}}-2 \bar{C}$ is skew symmetric.

This can be proven in the same way as Lemma 2 .

\section{Dynamic control of head position}

We have first applied an exact linearization method to control the final value of $w$ using Model I. However, the undulation of the robot was very small which resulted in very large input torques.

Therefore, we employ a PD control based on the Lyapunov function method on Model $I$ in (12) and investigate the shape of the trace. The candidate for the Lyapunov function is proposed as

$$
V=\frac{1}{2} \dot{w}^{T} \tilde{M} \dot{w}+\frac{1}{2}(w-r)^{T} B_{0}(w-r) .
$$

In this equation, $r$ is a constant position reference of $w$; $B_{0}>0$ is a design parameter. We use Lemma 2 and avoid taking the inversion of $E^{T} F$ as much as possible to obtain the derivative of $V$ as 

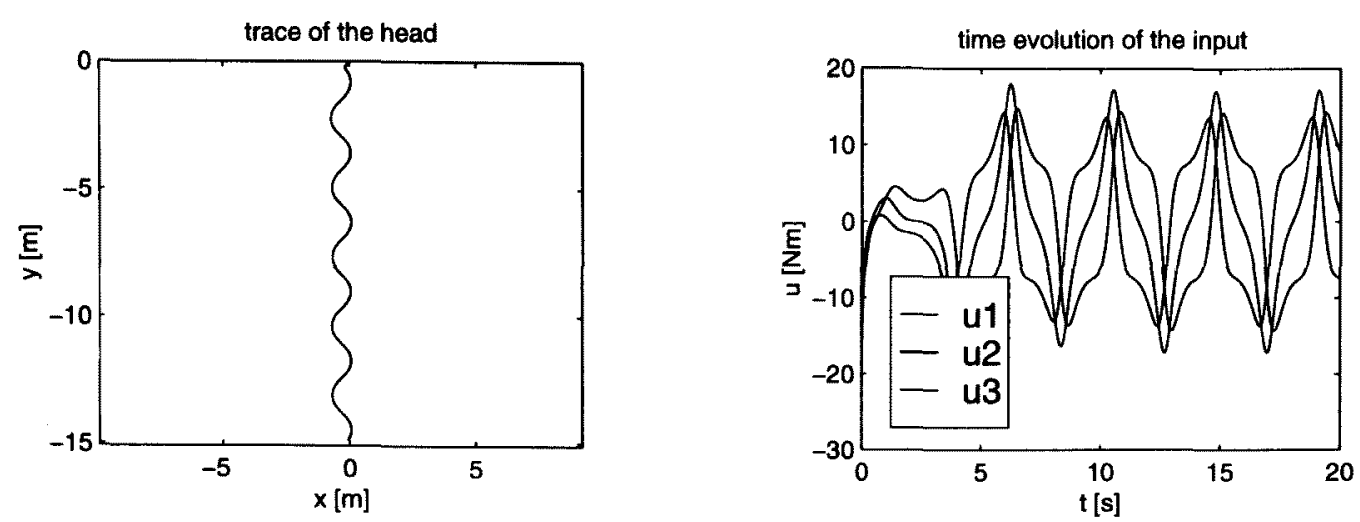

Fig. 3. Simulation of motion of a four-link snake robot controlled with fixed control error

$$
\begin{aligned}
& \dot{V}=\dot{w}^{T} \tilde{M} \ddot{w}+\frac{1}{2} \dot{w}^{T} \dot{\tilde{M}} \dot{w}+\dot{w}^{T} B_{0}(w-r) \\
& =\dot{w}^{T}\left[-\tilde{C} \dot{w}-\tilde{D} \dot{w}-\tilde{U}+F^{T} E u+\frac{1}{2} \dot{\tilde{M}} \dot{w}+B_{0}(w-r)\right] \\
& =\dot{w}^{T}\left[-\tilde{D} \dot{w}-\tilde{U}+F^{T} E u+B_{0}(w-r)\right] \\
& =-\dot{w}^{T}\left(\tilde{D}+F^{T} E A_{0} E^{T} F\right) \dot{w}<0,
\end{aligned}
$$

providing the control $u$ is given in a form

$$
u=-A_{0} E^{T} F \dot{w}-\left(F^{T} E\right)^{\dagger}\left(B_{0}(w-r)-\tilde{U}\right), \cdots
$$

where $A_{0} \geq 0$ is another design parameter.

We have started the investigation of the motion with a three link robot ${ }^{(6)}$. As a result, the robot undulated and the head converged to the reference point $r$. However, such a robot takes singular postures periodically which requires infinite magnitude of the inputs. Therefore, we analyzed the singular postures, which can occur when the body is not folded, to obtain:

Lemma 4 When $p_{i} \neq \pm \pi(\forall i=1 \sim n-1)$, matrix $E^{T} F(n-1 \times 2)$ drops rank if and only if

$$
p_{1}=p_{2}=\ldots=p_{n-1} \text {. }
$$

See Appendix for proof.

It follows from this lemma that the three-link robot cannot avoid singular postures since the line $p_{1}=p_{2}$ divides the plane $p \in R^{2}$ and the pair of relative angles $\left(p_{1}, p_{2}\right)$ of the robot inevitably crosses the line in order to realize symmetrical undulatory motion. Therefore, we conclude that we need at least four links to produce a snake robot which can move forward without taking singular postures.

Fig. 2 shows the simulation results for a four-link robot, where the parameters are given by

$$
\begin{aligned}
& m=1(\mathrm{~kg}), l=0.5(\mathrm{~m}), D_{\theta}=0, \quad D_{p}=0, \\
& D_{x y}=10\left(\mathrm{kgsm}^{-1}\right), \alpha=0(\mathrm{deg}), \beta=0(\mathrm{deg}), \\
& A_{0}=10, \quad B_{0}=20, \quad r=(0,-15)^{T}(\mathrm{~m}),
\end{aligned}
$$

and the simulation was carried out for $t=0 \sim 20 \mathrm{~s}$. The robot undulates to asymptotically reach the reference and the trace of the head may be approximated by a serpenoid curve. We can observe that the undulatory motion becomes slower as it approaches the reference
point.We can also show that the period of the undulatory motion becomes longer if $B_{0}$ is smaller.

Nevertheless, the inputs, even finite, are not smooth and contain peaks. In order to realize a snake robot that uses inputs of uniform amplitude over the whole control period, we have introduced a penalty into (20) as

$$
\begin{aligned}
V & =\frac{1}{2 k} \frac{C_{0}}{\left(\theta^{T} E H H^{T} E^{T} \theta\right)^{k}}+\frac{1}{2} \dot{w}^{T} \tilde{M} \dot{w} \\
& +\frac{1}{2}(w-r)^{T} B_{0}(w-r),
\end{aligned}
$$

where the first term prevents $p$ from taking singular postures since

$$
\begin{aligned}
& \delta p=\left(p_{2}-p_{1}, \ldots, p_{n}-p_{n-1}\right)^{T}=H^{T} p=H^{T} E^{T} \theta \\
& H^{T}=\left[\begin{array}{cccc}
-1 & 1 & & \\
& : & & \\
& & -1 & 1
\end{array}\right] \quad(\overline{n-2} \times \overline{n-1})
\end{aligned}
$$

holds. We can obtain a similar control formula as (21), however, it does not work very effectively. Instead, increasing the number of links makes the inputs smaller and without large peaks. However, this time, the amplitude of the undulatory motion tends to decrease.

On the other hand, when the velocity of the head is controlled to be a constant, the robot becomes straight from the following lemma.

Lemma 5 When $\dot{w}=v=\left(v_{x}, v_{y}\right)^{T}=$ const with $v_{y}<0$, then $\bar{\theta}_{1}$ satisfying $\tan \left(\bar{\theta}_{1}\right)=\frac{v_{x}}{v_{y}}$ is a stable equilibrium. Furthermore, with $\mathrm{C} \bar{\theta}_{1}>0, p_{i}=0$ are also stable equilibriums.

\section{See Appendix for proof.}

Therefore, in order to make the magnitude of input uniformly small during the motion, it is better not to give a definite reference from the beginning of the motion.

Simulation of the motion of the robot, when the controlling error $e=r-w$ is fixed as $e=(0,-3.5)^{T}=r_{0}$ is shown in Fig. 3. We can see that the magnitude of the input reduces to $30 \%$, while the robot travels the same distance as before. By choosing $r_{0}$, we can adjust the amplitude and frequency of the undulatory motion. 


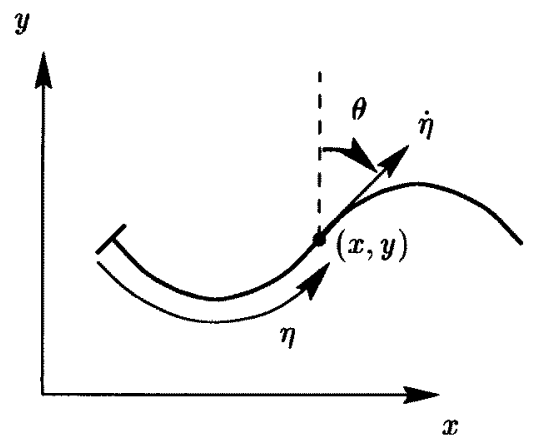

Fig. 4. Length and direction angle of a curve

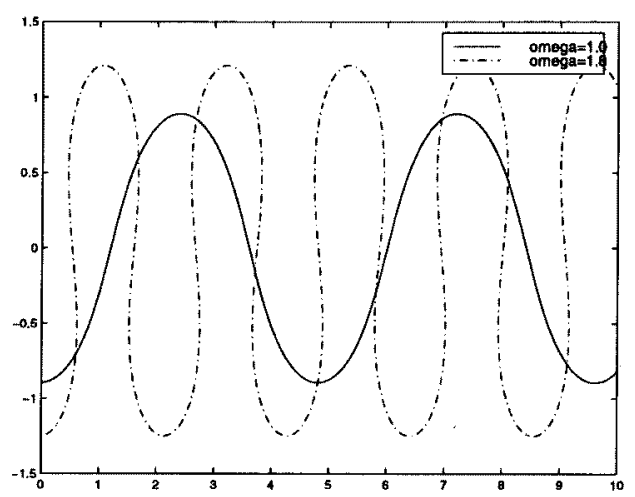

Fig. 5. Examples of a serpenoid curve $\left(\omega_{1}=1.0, k_{1}=1\right.$, $\omega_{2}=1.8, k_{2}=2.5$ )

\section{Introducing a serpenoid trace}

Assuming that snakes have developed an efficient way of creeping, we look back to nature for inspiration. To describe the undulation of natural snakes, Hirose ${ }^{(1)}$ has proposed a serpenoid curve. Thus, in order to minimize the energy needed for motion of our snake robot, we restrict the motion to some kind of a serpenoid curve.

An arbitrary curve can be described by its length $\eta$ measured from some reference point and the direction angle $\theta(\eta)$, as shown in Fig. 4. Denote a small deviation of $\eta$ as $d \eta$. Since it holds

$$
d x=\sin \theta d \eta, \quad d y=\cos \theta d \eta,
$$

we can describe the relation between $(x, y)$ and $\eta$ as

$$
\begin{aligned}
& \frac{d x}{d \eta}=\sin \theta, \quad \frac{d y}{d \eta}=\cos \theta \\
& \dot{x}=\frac{d \eta}{d t} \frac{d x}{d \eta}=\dot{\eta} \sin \theta, \quad \dot{y}=\frac{d \eta}{d t} \frac{d y}{d \eta}=\dot{\eta} \cos \theta \\
& \eta(t)=\int_{0}^{t} \sqrt{\dot{x}^{2}+\dot{y}^{2}} d t .
\end{aligned}
$$

Following Hirose ${ }^{(1)}$, the serpenoid curve is defined as to satisfy

$$
\frac{d x}{d \eta}=\sin (\omega \sin (k \eta)), \quad \frac{d y}{d \eta}=\cos (\omega \sin (k \eta))
$$

From this definition and relations (23), it is easy to see that

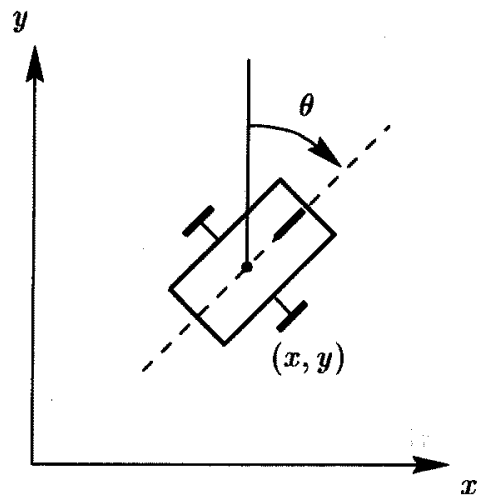

Fig. 6. Two-wheel cart

$$
\theta=\omega \sin (k \eta) .
$$

Therefore, the serpenoid function is a curve whose direction angle varies sinusoidally along the distance and shape of the curve depends on the the values of $\omega, k$ as shown in Fig. 5. By integrating (24) we get the position of any point on the curve as

$$
\begin{aligned}
& x(\eta)=\int_{0}^{\eta} \sin (\omega \sin (k r)) d r, \\
& y(\eta)=\int_{0}^{\eta} \cos (\omega \sin (k r)) d r .
\end{aligned}
$$

4.1 Two-wheel cart and serpenoid trace Before constraining the trace of the motion of the snake robot consider a problem where the trace of the COM of a two-wheel cart is controlled to follow the serpenoid curve.

As it is well known, the two-wheel cart is a nonholonomic system ${ }^{(2)(5)}$. Here we impose an additional holonomic constraint by a control so as to change the nonholonomic constraints to be integrable such that they become an invariant manifold.

As in Fig. 6, the pair $(x, y)$ denotes the position of the COM, $\theta$ the orientation of the cart measured from the $y$ axis and $\eta$ length of the trace of the COM. Since the wheels cannot slip sideways, we have

$$
\dot{x} \mathrm{C} \theta-\dot{y} \mathrm{~S} \theta=0
$$

as a non-integrable velocity constraint. The front castor maintains balance and its effect will be ignored.

The velocity of the trace length $\dot{\eta}$ satisfies

$$
\dot{x} \mathrm{~S} \theta+\dot{y} \mathrm{C} \theta=\dot{\eta} .
$$

We introduce the following holonomic constraint (control constraint)

$$
\theta(t)=\omega \sin (k \eta(t))
$$

which will be realized by control. Substituting (28) into the derivative of (29) we obtain another velocity constraint as

$$
\dot{\theta}=\omega k \cos (k \eta) \dot{\eta}=\omega k \cos (k \eta)(\dot{x} \mathrm{~S} \theta+\dot{y} \mathrm{C} \theta) .
$$

The Pfaffian form of the velocity constraints (27) and (30) becomes 

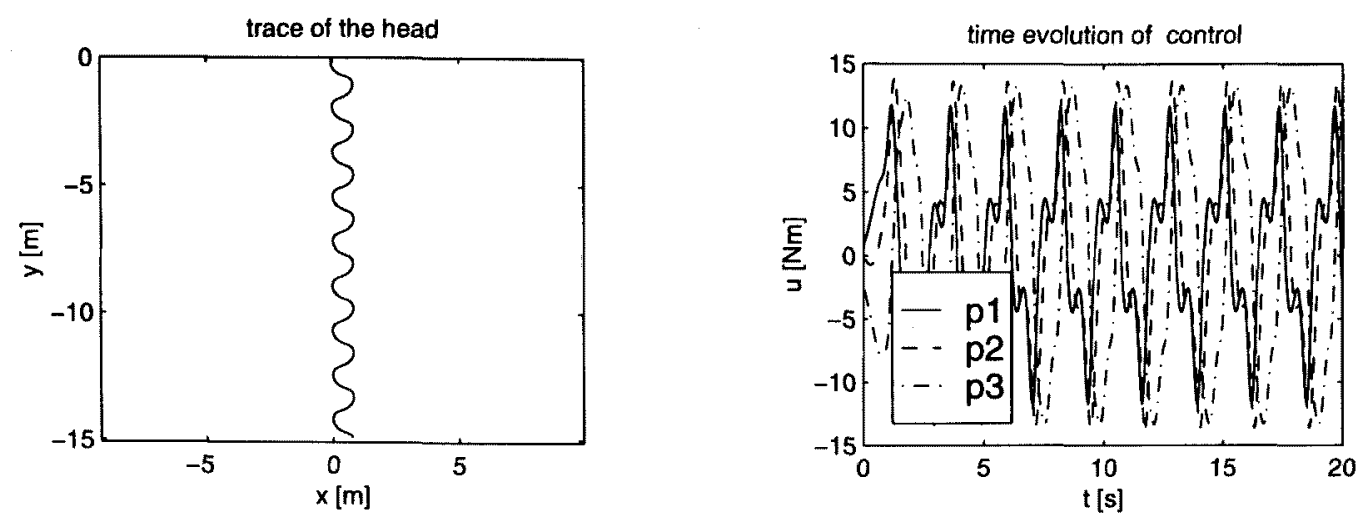

Fig. 7. Motion of a four-link snake robot controlled with fixed control error, constrained to a holonomic manifold

$$
\left[\begin{array}{ccc}
0 & \mathrm{C} \theta & -\mathrm{S} \theta \\
-\frac{1}{\omega k \cos (k \eta)} & \mathrm{S} \theta & \mathrm{C} \theta
\end{array}\right] \dot{q}=0 ; \quad q=\left[\begin{array}{l}
\theta \\
x \\
y
\end{array}\right]
$$

The Pfaffian constraint has one dimensional null space for any position, which means that it is involutive ${ }^{(5)}$. Hence the velocity constraints are integrable and give the first integral

$$
f(q)=c: \quad c \text { is constant }
$$

which satisfies

$$
\frac{d f}{d t}=\frac{\partial f}{\partial q} \dot{q}=0
$$

This implies that $f=c$ constructs an invariant manifold and once the trajectory of the motion reaches this manifold it is kept there as long as the control constraint is preserved.

In the present case, we solve the constraints (27) and (28) for $\dot{x}$ and $\dot{y}$ to get

$$
\begin{aligned}
& \dot{x}=\dot{\eta} \sin (\theta) \\
& \dot{y}=\dot{\eta} \cos (\theta)
\end{aligned}
$$

Substituting for $\theta$ from (29) and integrating yields

$$
f(q)=\left[\begin{array}{l}
x-\int_{0}^{\eta} \sin (\omega \sin (k r)) d r \\
y-\int_{0}^{\eta} \cos (\omega \sin (k r)) d r
\end{array}\right]=\left[\begin{array}{l}
c_{1} \\
c_{2}
\end{array}\right]
$$

as the first integral of (31) and hence the serpenoid curve as a trace of the COM.

4.2 Snake robot and serpenoid trace If we impose the control constraint on the first link of the $n$-link snake robot in Fig. 1 , the set of $n$ velocity constraints (4) becomes holonomic and the snake robot, with $(n+2)$ degrees of freedom is represented by system with one degree of freedom on one invariant manifold.

Let the orientation of the head be constrained by

$$
\theta_{1}=\omega \sin \left(k \eta_{1}\right) \text {. }
$$

Then we have the serpenoid trace

$$
\begin{aligned}
& x_{1}=\int_{0}^{\eta_{1}} \sin (\omega \sin (k r)) d r+c_{1}, \\
& y_{1}=\int_{0}^{h_{1}} \cos (\omega \sin (k r)) d r+c_{2}
\end{aligned}
$$

as an invariant motion of the COM of the first link which together with (14) shows that the tip $(x, y)$ of the head also draws a serpenoid curve.

When (35) holds, using transformation matrix $G$ defined in (15), the velocity constraints that do not contain $\dot{\theta}_{1}$ any more have a form

$$
\begin{aligned}
\dot{p} & =E^{T} F \dot{w}=E^{T} F G \cdot G \dot{w}=E^{T} F G\left[\begin{array}{c}
l \dot{\theta}_{1} \\
\dot{\eta}_{\mathbf{1}}
\end{array}\right] \\
& =E^{T} F G\left[\begin{array}{c}
k \omega l \cos \left(k \eta_{1}\right) \\
1
\end{array}\right] \dot{\eta}_{1}
\end{aligned}
$$

which is equivalent to

$$
\frac{d p}{d \eta_{1}}=E^{T} F G\left[\begin{array}{c}
k \omega l \cos \left(k \eta_{1}\right) \\
1
\end{array}\right]
$$

where $E^{T} F G$ is a function of $p$. Therefore, the constraints are integrable.

We consider a control of the snake robot where the control constraint (35) and a command which directs the robot forward are taken into account. The robot model for a distance control is given by Model II (19). The control constraint and distance control with a reference distance along the curve $\gamma$

$$
\begin{aligned}
& F_{1}=l \theta_{1}-l \omega \sin \left(k \eta_{1}\right)=0, \\
& F_{2}=\eta_{1}-\gamma=0,
\end{aligned}
$$

can be realized by the zeroing method as follows ${ }^{(9)}$.

The differential equations

$$
\begin{aligned}
& \ddot{F}_{1}+\alpha \dot{F}_{1}+\beta F_{1}=0, \\
& \ddot{\eta}_{1}+\vartheta \dot{\eta}_{1}+\xi\left(\eta_{1}-\gamma\right)=0
\end{aligned}
$$

where

$$
\begin{aligned}
& \dot{F}_{1}=l \dot{\theta}_{1}-l \omega k \cos \left(k \eta_{1}\right) \dot{\eta}_{1} \\
& \ddot{F}_{1}=l \ddot{\theta}_{1}-l \omega k \cos \left(k \eta_{1}\right) \ddot{\eta}_{1}+l \omega k^{2} \sin \left(k \eta_{1}\right) \dot{\eta}_{1}^{2}
\end{aligned}
$$

and $\alpha, \beta, \vartheta, \xi$ are positive coefficients, determine the dynamics of convergence of the trace shape to the serpenoid curve and of the distance $\eta_{1}$ to the given distance, respectively.

Define a nonsingular transformation matrix 


$$
P=\left[\begin{array}{cc}
1 & -l \omega k \cos \left(k \eta_{1}\right) \\
0 & 1
\end{array}\right]
$$

and multiply the vector $\left[\begin{array}{c}l \ddot{\theta}_{1} \\ \ddot{\eta}_{1}\end{array}\right]$ by $P$ from the left to get

$$
P\left[\begin{array}{l}
l \ddot{\theta}_{1} \\
\ddot{\eta}_{1}
\end{array}\right]=\left[\begin{array}{l}
l \ddot{\theta}_{1}-l \omega k \cos \left(k \eta_{1}\right) \ddot{\eta}_{1} \\
\ddot{\eta}_{1}
\end{array}\right]:=K .
$$

Substituting from (41) and (40) into (43) yields

$$
\begin{aligned}
& {\left[\begin{array}{c}
l \ddot{\theta}_{1} \\
\ddot{\eta}_{1}
\end{array}\right]=P^{-1} K} \\
& =P^{-1}\left[\begin{array}{c}
-l \omega k^{2} \sin \left(k \eta_{1}\right) \dot{\eta}_{1}^{2}-\alpha \dot{F}_{1}-\beta F_{1} \\
-\vartheta \dot{\eta}_{1}-\xi\left(\eta_{1}-\gamma\right)
\end{array}\right] .
\end{aligned}
$$

Next, we substitute (44) into Model II (19) and obtain

$$
\bar{M} P^{-1} K+(\bar{C}+\bar{D})\left[\begin{array}{c}
l \dot{\theta}_{1} \\
\dot{\eta}_{1}
\end{array}\right]+\bar{U}=G F^{T} E u .
$$

Thus if we choose the control as

$$
u=\left(G F^{T} E\right)^{\dagger}\left[(\bar{C}+\bar{D})\left[\begin{array}{c}
l \dot{\theta}_{1} \\
\dot{\eta}_{1}
\end{array}\right]+\bar{U}+\bar{M} P^{-1} K\right] \text {, }
$$

the motion of the robot described by Model II (19) will satisfy (44). This ensures that $F_{1} \rightarrow 0$ and $F_{2} \rightarrow 0$.

The parameters $\omega, k$ of the serpenoid curve are determined so as to minimize the magnitude of input torques required for the creeping motion. They are optimal in the sense that any other choice would result in bigger torque amplitude. Fig. 7 shows the simulation results for a four link robot controlled with the error being fixed to a constant value $\gamma=-1.4$ so that the robot travels with the same velocity as in Fig. 3 . We can see that the input magnitude is reduced to about $70 \%$ of the input in Fig. 3.

Although increasing the number of links further reduces the input requirements, the undulation becomes smaller too, as can be noticed from 4.2 of the optimal values of parameters $\omega$ and $k$ for robots with different number of links $n$.

Table 1. Optimal parameters of the serpenoid curve

\begin{tabular}{c|c|c}
\hline number of links & $\omega$ & $k$ \\
\hline$n=4$ & 0.76 & 3.2 \\
$n=6$ & 0.61 & 4.8 \\
$n=10$ & 0.35 & 8.4 \\
\hline
\end{tabular}

4.3 Path tracking using serpenoid trace We would also like to address the topic of tracking a given path here. Since the undulation is an essential characteristic of the snake robot movement, the usual tracking algorithms, such as ${ }^{(7)(8)}$, cannot be applied directly. However, since we can constrain the nonholonomic system to a one dimensional manifold, we can make the snake moving in the desired direction by changing the manifold.

Suppose that the reference position $w_{r}=\left(x_{r}, y_{r}\right)$ and

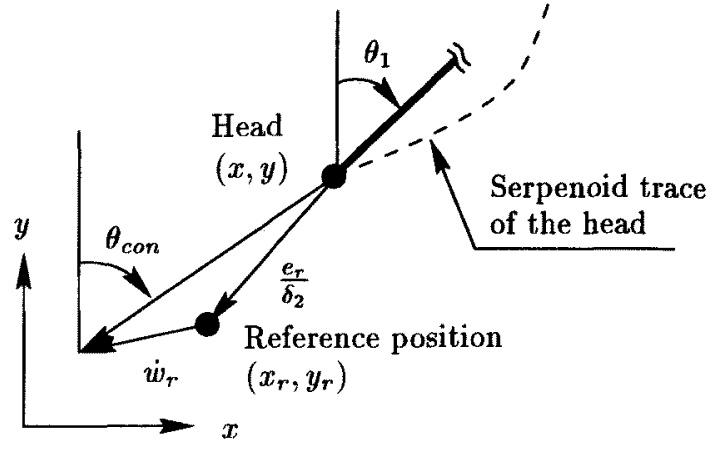

Fig. 8. Control angle $\theta_{\text {con }}$ for $\delta_{2}=1$

velocity $\dot{w}_{r}=\left(\dot{x}_{r}, \dot{y}_{r}\right)$ of the head are available. This information may be provided by a path planner ${ }^{(8)}$. The current position $w=(x, y)$ of the head can be obtained via odometry.

First, we constrain the motion of the robot to a serpenoid curve where we substitute the optimal values of parameters $\omega, k$ that minimize the inputs magnitudes (4.2). In the next step we need to adjust the direction in which the snake undulates according to the reference and current position of the head, and to the direction of the reference velocity. Let us denote this control angle by $\theta_{c o n}$. Finally, the command that directs the robot forward must correspond to a magnitude of the reference velocity and to the distance from the head to the reference position.

According to these consideration the constraints (39) are rewritten as

$$
\begin{aligned}
& F_{1}=l \theta_{1}-l \omega \sin \left(k \eta_{1}\right)+\theta_{\text {con }}=0, \\
& F_{2}=\dot{\eta}_{1}-\left(\left\|\dot{w}_{r}\right\|_{2}+\delta_{1} e_{r}\right)=0 .
\end{aligned}
$$

As shown in Fig. 8, the control angle $\theta_{\text {con }}$, added to the first control constraint, is computed from a direction of the reference velocity and the direction from the head to the reference position. This may be formally written as

$$
\theta_{c o n}=\operatorname{atan} 2\left(\left[\left(y_{r}-y\right)+\delta_{2} \dot{y}_{r}\right],\left[\left(x_{r}-x\right)+\delta_{2} \dot{x}_{r}\right]\right),
$$

where the function $\operatorname{atan} 2(y, x)$ stands for a four quadrant inverse tangent function and $\delta_{2}$ is a positive control parameter.

The velocity of the snake is determined by the second constraint (47), where $e_{r}$ is a distance between the current and reference position of the robot's head, defined as follows

$$
e_{r}=\left(x_{r}-x\right) \sin \left(\theta_{c o n}\right)+\left(y_{r}-y\right) \cos \left(\theta_{c o n}\right) \cdots
$$

and $\delta_{1}$ is a positive control parameter.

Using the zeroing method in the same way as in the previous subsection, we obtain a control law similar to (46). The robot then follows the reference trajectory as shown in Fig. 9.

\section{Conclusion}

Considering a snake-like robot consisted of rigid links 

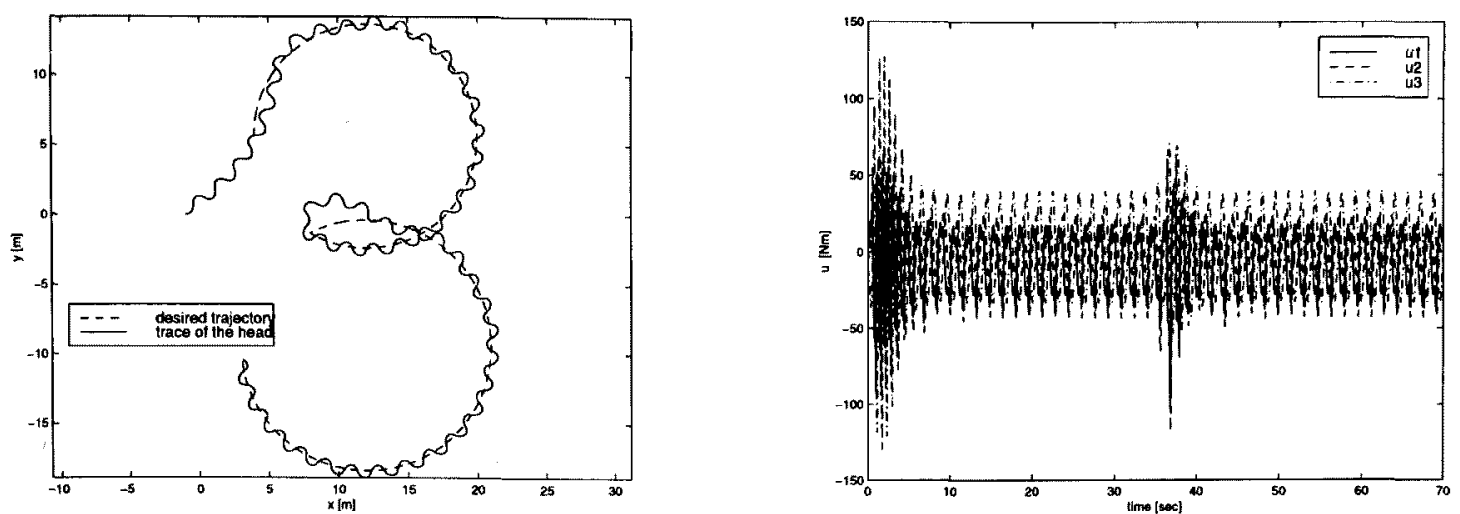

Fig. 9. Simulation of a four-link snake robot following a path with given velocity

and free wheels, we have developed several control methods based either on Lyapunov method or on variable constrained control. Further on, we have focused on minimizing the magnitude of the input torque required for traveling with prescribed velocity. The benefit of constraining a nonholonomic system to a onedimensional manifold was demonstrated on a simple tracking example. We are now carrying out experiments with a mechanical model of the snake robot. Further steps will include reformulating the nonholonomic nonslipping constraints so that the normal force acting on the wheel must not exceed force arising from friction with ground.

\section{Acknowledgment}

This research has been supported by TiTech COE Super-Mechano-System Grant. We would like to thank the members of the snake research group of the COE and especially $H$. Yamauchi for their help.

(Manuscript received May 26, 1999, revised Oct., 29, 1999)

\section{References}

(1) S. Hirose: Biologically Inspired Robots: Snake-Like Locomotors and Manipulators (transl.) Oxford, Oxford University Press (1993)

(2) J. Ostrowski and J. Burdick: "The Geometric Mechanics of Undulatory Robotic Locomotion", Int. J. of Robotics Research, 17, No.7, 683-701 (1998)

(3) C. Samson: "Control of Chained Systems Application to Path Following and Time-Varying Point Stabilization of Mobile Robots", IEEE Trans. on Autom. Control, 40, No. 1, 64-77 (1995)

(4) D. T. Greenwood: Classical Dynamics, Prentice Hall (1977)

(5) R. M. Murray, Z. Li and A. S. Sastry: A Mathematical Introduction to Robotic Manipulation, CRC Press, (1993)

(6) P. Prautsch: "Control and Analysis of a Gait of Snake Robot", Report of IEEJ, Industrial Application Research Group, IIC-90-10 (1999)

(7) Y. Kanayama, Y. Kimura, F. Miyazaki, T. Noguchi: "A Stable Tracking Control Method for a Non-Holonomic Mobile Robot", Proc. IEEE/RSJ International Workshop on Intelligent Robots and Systems, 1236-1241 (1991)

(8) W. Nelson, I. Cox: "Local Path Control for an Autonomous Vehicle", Proc. IEEE Conf. on Robotics and Automation, 1504-1510, 1988
(9) T. Ikeda, T.K. Nam, X. Xin, T. Mita: "Nonholonomic Variable Constraint Control of Free Flying Robots and Its Convergence", Proc. of 28th SICE Symposium on Control Theory (1999)

\section{Appendix}

Proof of Lemma 2

It can readily be seen that $\dot{M}_{22}=0$ and thus

$$
\begin{aligned}
& \dot{\tilde{M}}=\dot{F}^{T} M_{11} F+F^{T} M_{12} \dot{F}+\dot{F}^{T} M_{12} \\
& +M_{12}^{T} \dot{F}+F^{T} \dot{M}_{11} F+F^{T} \dot{M}_{12}+\dot{M}_{12}^{T} F .
\end{aligned}
$$

Then compute

$$
\begin{aligned}
& \dot{\tilde{M}}-2 \tilde{C}=\left(\dot{F}^{T} M_{11} F-F^{T} M_{11} \dot{F}\right) \\
& +\left(\dot{F}^{T} M_{12}-M_{12}^{T} \dot{F}\right)+F^{T}\left(\dot{M}_{11}-2 C_{1}\right) F+\gamma,
\end{aligned}
$$

where

$$
\gamma=F^{T} \dot{M}_{12}+\left(\dot{M}_{12}^{T}-2 C_{2}\right) F .
$$

According to Lemma 1, the third term term in (A2) is skew symmetric and thus we only need to show that $\gamma$ is skew symmetric too. However, that follows from the second part of Lemma 1 and thus $\dot{M}-2 \tilde{C}$ is skew symmetric.

\section{Proof of Lemma 4}

Rewrite the equation (5) in the term of relative angle $p$ and absolute angle of the first link $\theta_{1}$ as

$$
\begin{aligned}
& {\left[\begin{array}{c}
l \\
l a_{12} \\
l a_{13} \\
\vdots \\
l a_{1 n}
\end{array}\right] \dot{\theta_{1}}+\left[\begin{array}{cccc}
0 & & \\
l & & \\
l a_{23} & l & & \\
\vdots & & \ddots & \\
l a_{2 n} & \ldots & l a_{(n-1) n} & l
\end{array}\right] \dot{p}} \\
& =\left[\begin{array}{cc}
-\mathrm{C} \theta_{1} & \mathrm{~S} \theta_{1} \\
-\mathrm{C} \theta_{2} & \mathrm{~S} \theta_{2} \\
\vdots & \vdots \\
-\mathrm{C} \theta_{n} & \mathrm{~S} \theta_{n}
\end{array}\right] \dot{w}, \ldots \ldots \ldots \ldots \ldots
\end{aligned}
$$

where $a_{i j}=2 \sum_{k=i}^{(j-1)} C_{k j}+1$ and we have used the relation between absolute angles $\theta$ and relative angles $p$. Next substitute for $\dot{\theta}_{1}$ from the first row. After deleting the first row we obtain 


$$
\begin{aligned}
& l\left[\begin{array}{cccc}
1 & & & \\
a_{23} & 1 & & \\
\vdots & & \ddots & \\
a_{2 n} & \ldots & a_{(n-1) n} & 1
\end{array}\right] \dot{p} \\
& =\left[\begin{array}{cc}
-\mathrm{C} \theta_{2}+a_{12} \mathrm{C} \theta_{1} & \mathrm{~S} \theta_{2}-a_{12} \mathrm{~S} \theta_{1} \\
-\mathrm{C} \theta_{3}+a_{13} \mathrm{C} \theta_{1} & \mathrm{~S} \theta_{3}-a_{13} \mathrm{~S} \theta_{1} \\
\vdots & \vdots \\
-\mathrm{C} \theta_{n}+a_{1 n} \mathrm{C} \theta_{1} & \mathrm{~S} \theta_{n}-a_{1 n} \mathrm{~S} \theta_{1}
\end{array}\right] \dot{w}
\end{aligned}
$$

or, shortly,

$$
A \dot{p}=B \dot{w} ; \quad \dot{p}=A^{-1} B \dot{w} .
$$

Since the matrix $A$ is nonsingular for any $\theta_{i}$ and thus also for any $p$, we only need to analyze the matrix $B$. Rank of a matrix does not change after multiplying by any nonsingular matrix, so we multiply $B$ by transformation matrix $G$ defined in (15) from the right and analyze the rank of

$$
\hat{B}_{n}=\left[\begin{array}{cc}
C_{21}-a_{12} & S_{21} \\
C_{31}-a_{13} & S_{31} \\
\vdots & \vdots \\
C_{n 1}-a_{1 n} & S_{n 1}
\end{array}\right]
$$

instead. Notice that $\hat{B}_{n}$ does not contain $\theta_{1}$ and its rank thus depend only on relative angles, i.e. singular postures depend on the robot's shape, not the orientation. The statement of Lemma 4 will be proven by induction for any number of links $n \geq 3$.

- For $n=3$, the determinant of matrix $\hat{B}_{3}$

$$
\hat{B}_{3}=\left[\begin{array}{ll}
C_{21}-\left(2 C_{12}+1\right) & S_{21} \\
C_{31}-\left(2 C_{13}+2 C_{23}+1\right) & S_{31}
\end{array}\right]
$$

is after some calculation

$$
\begin{aligned}
\left|\hat{B}_{3}\right| & =S_{21}\left(C_{31}+1+2 C_{23}\right)-S_{31}\left(C_{21}+1\right) \\
& =\sin \left(p_{1}\right)\left(\cos \left(p_{1}+p_{2}\right)+1+2 \cos \left(p_{2}\right)\right) \\
& -\sin \left(p_{2}+p_{3}\right)\left(\cos \left(p_{1}\right)+1\right) \\
& =\sin \left(p_{1}\right)-\sin \left(p_{2}\right)+\sin \left(p_{1}\right) \cos \left(p_{2}\right) \\
& -\sin \left(p_{2}\right) \cos \left(p_{1}\right) \ldots \ldots \ldots \ldots \ldots \ldots \ldots \ldots \ldots \ldots \ldots \ldots
\end{aligned}
$$

Now we look for $p$ such that $\left|\hat{B}_{3}\right|=0$. It can be readily seen that for $\left(p_{1} \neq \pm \pi, p_{2} \neq \pm \pi\right)$ we can solve an equation

$$
\frac{\sin \left(p_{1}\right)}{1+\cos \left(p_{1}\right)}=\frac{\sin \left(p_{2}\right)}{1+\cos \left(p_{2}\right)}
$$

to get

$$
p_{1}=p_{2} \quad \text { and } \quad\left(p_{1} \neq \pm \pi, p_{2} \neq \pm \pi\right) .
$$

- Suppose that Lemma 4 is valid for some number of links $(k-1) \geq 3$. Then we do not need to compute the whole determinant of the matrix $\hat{B}_{k}$

$$
\hat{B}_{k}=\left[\begin{array}{ll}
C_{21}-\left(2 C_{12}+1\right) & S_{21} \\
C_{31}-\left(2 C_{13}+2 C_{23}+1\right) & S_{31} \\
\vdots & \vdots \\
C_{k 1}-\left(2 C_{1 k}+2 C_{2 k}+\ldots+1\right) & S_{k 1}
\end{array}\right]
$$

since $\operatorname{rank} \hat{B}_{k}=1$ for $p=p_{1}=p_{2}=\ldots=p_{(k-2)} \neq \pm \pi$. Thus in order to obtain the value of $p_{k-1}$ for which the whole matrix $\hat{B}_{k}$ drops rank, it is sufficient to calculate a determinant of a submatrix constructed by adding an arbitrary row $i, i<k-1$ to the $k-1$ th one. We will use the first row and compute the determinant

$$
\begin{aligned}
\left|B_{k}^{\prime}\right| & =S_{21}\left(C_{1 k}+1+2 C_{2 k}+2 C_{3 k}+\ldots\right. \\
& \left.+2 C_{(k-1), k}\right)-S_{k 1}\left(C_{12}+1\right) \\
& =\cos \left((k-2) p+p_{k-1}\right) \sin (p) \\
& -\sin \left((k-2) p+p_{k-1}\right) \cos (p) \\
& -\sin \left((k-2) p+p_{k-1}\right) \\
& +\sin (p)+2 \cos \left((k-3) p+p_{k-1}\right) \sin (p) \\
& +2 \cos \left((k-4) p+p_{k-1}\right) \sin (p)+\ldots \\
& -2 \cos \left(p_{k-1}\right) \sin (p) \\
& =\sin (p)-\sin \left(p_{k-1}\right)+\sin (p) \cos \left(p_{k-1}\right) \\
& -\cos (p) \sin \left(p_{k-1}\right) \ldots \ldots \ldots \ldots \ldots \ldots \ldots
\end{aligned}
$$

It can be seen that $\left|B_{k}^{\prime}\right|=0$ for

$$
p=p_{1}=p_{2}=\ldots=p_{k-1}(p \neq \pm \pi) .
$$

- Since Lemma 4 is valid for $n=k$ provided it is valid for $n=k-1$ and we have proven it for $n=3$ we conclude that it is valid for all $n \geq 3$.

\section{Proof of Lemma 5}

We need to analyze

$$
\begin{aligned}
\dot{\theta}_{1} & =\frac{1}{l}\left(-\mathrm{C} \theta_{1}, \mathrm{~S} \theta_{1}\right) \dot{w}, \ldots \ldots \ldots \ldots \ldots \ldots \\
\dot{p} & =E^{T} F G \cdot G \dot{w}=E^{T} F_{A}^{-1} F_{B} G . G \dot{w}, \cdots
\end{aligned}
$$

where matrix $G$ was defined in (15) and $\dot{w}=\left(v_{x}, v_{y}\right)^{T}=$ const.

As for (A11), the equilibrium $\bar{\theta}_{1}$ must satisfy $\left(-\mathrm{C} \bar{\theta}_{1}, \mathrm{~S} \bar{\theta}_{1}\right)$ from which we get $\tan \left(\bar{\theta}_{1}\right)=\frac{v_{x}}{v_{y}}$. Since $e_{1}=\theta_{1}-\bar{\theta}_{1}$ satisfies

$$
\dot{e}_{1}=\frac{1}{l}\left(\mathrm{C} \bar{\theta}_{1}, \mathrm{~S} \bar{\theta}_{1}+e_{1}\right) \dot{w}=\frac{v_{y}}{l} e_{1}
$$

by the first approximation, $\bar{\theta}_{1}$ is stable, provided $v_{y}<0$.

Now we analyze (A12). First we compute

$$
\begin{aligned}
G \dot{w} & =\left[\begin{array}{l}
0 \\
\mathrm{~S} \vec{\theta}_{1} v_{x}+\mathrm{C} \bar{\theta}_{1} v_{y}
\end{array}\right]=\left[\begin{array}{l}
0 \\
1
\end{array}\right] v_{h} ; \cdots \\
v_{h} & =\frac{v_{y}}{\mathrm{C} \bar{\theta}_{1}}
\end{aligned}
$$

when $\theta=\bar{\theta}_{1}$. Next, in (A12), since

$$
\begin{aligned}
& F_{B} G\left[\begin{array}{l}
0 \\
1
\end{array}\right]=\left[\begin{array}{cc}
1 & 0 \\
\cos \left(p_{1}\right) & \sin \left(p_{1}\right) \\
\vdots & \vdots \\
\cos \left(p_{n-1}\right) & \sin \left(p_{n-1}\right)
\end{array}\right]\left[\begin{array}{l}
0 \\
1
\end{array}\right] \\
& =\left[\begin{array}{l}
0 \\
\sin \left(p_{1}\right) \\
\vdots \\
\sin \left(p_{n-1}\right)
\end{array}\right] \ldots \ldots \ldots \ldots \ldots \ldots \ldots \ldots \ldots \ldots
\end{aligned}
$$


and $E^{T} F_{A}^{-1}$ has the form

$$
\begin{aligned}
& E^{T} F_{A}^{-1}=\frac{1}{l}\left[\begin{array}{cccc}
* & & & \\
* & 1 & & \\
\vdots & * & \ddots & \\
* & * & * & 1
\end{array}\right] \\
& =\frac{1}{l}\left[\begin{array}{ccc}
* & 0 & \\
\hdashline * & C(p),
\end{array}\right] \ldots \ldots \ldots
\end{aligned}
$$

where $*$ stands for a combination of cosine functions of $p_{i}$, we get

$$
\dot{p}=\frac{C(p)}{l}\left[\begin{array}{l}
\sin p_{1} \\
\vdots \\
\sin p_{n-1}
\end{array}\right] v_{h}
$$

From (A17) we see that vector of relative angles $p$ satisfying $\sin \left(p_{i}\right)=k_{i} \pi$ is an equilibrium point. Using approximation $\cos \left(p_{i}\right)=1, \sin \left(p_{i}\right)=p_{i}$ around $p_{i}=0$ we get

$$
\dot{p}=\frac{v_{h}}{l} C(0) p .
$$

Since the eigenvalues of $C(0)$ are all equal to $1, p=0$ is a stable if and only if $v_{h}<0$ which together with condition $v_{y}<0$ yield $\mathrm{C} \bar{\theta}_{1}>0$.

Pavel Prautsch (Non-member) Was born in 1971. He re-

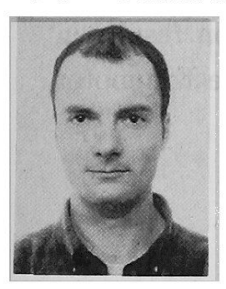
ceived his diploma from West Bohemian University, Pilsen, Czech Republic in 1994. He is currently working towards $\mathrm{Ph}$. D. degree at Tokyo Institute of Technology. His research interests include optimal control, robotics, mobile systems in particular.

Tsutomu Mita (Member) Was born in 1947. He received

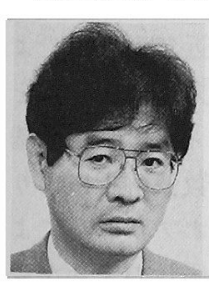
$\mathrm{Ph}$. D. degree in electrical engineering from Tokyo Institute of Technology in 1975. In the same year he became a Lecturer, then an Associate professor at Chiba University and in 1990 he became a Professor. Since April 1995 he is a Professor at Tokyo Institute of Technology. He is engaged in research and education of robotics and control theory. He won the best paper prizes from Sice in 1989 and 1994. He is author of 8 books in Japanese

Tetsuya Iwasaki (Member) Was born in 1964. He re-

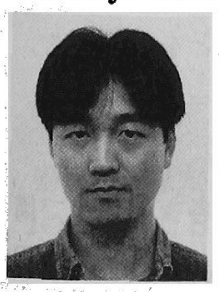
ceived the B.S. and M.S. degrees in electrical engineering from the Tokyo Institute of Technology in 1987 and 1990, respectively. He received the $\mathrm{Ph}$. $\mathrm{D}$. degree in aeronautics and astronautics from Purdue University, $W$ Lafayette, IN, USA in 1993. In 1995, he joined the Tokyo Institute of Technology, where he is currently an Associate Professor. His current research interests include modeling, robust and adaptive control and robotics. 